\title{
Numerical study of spin Hall transport in a two-dimensional hole-gas system
}

\author{
W. Q. Chen, ${ }^{1,2}$ Z. Y. Weng, ${ }^{1}$ and D. N. Sheng ${ }^{2}$ \\ ${ }^{1}$ Center for Advanced Study, Tsinghua University, Beijing, People's Republic of China 100084 \\ ${ }^{2}$ Department of Physics and Astronomy, California State University, Northridge, California 91330, USA \\ (Received 14 August 2005; revised manuscript received 17 October 2005; published 13 December 2005)
}

\begin{abstract}
We present a numerical study of the spin Hall effect in a two-dimensional hole gas system in the presence of disorder. We find that the spin Hall conductance (SHC), extrapolated to the thermodynamic limit, remains finite in a wide range of disorder strengths for a closed system on torus. But there is no intrinsic spin Hall accumulation as induced by an external electric field once the disorder is turned on. The latter is examined by performing a Laughlin's Gedanken gauge experiment numerically with the adiabatic insertion of a flux quantum in a belt-shaped sample, in which the absence of level crossing is found under the disorder effect. Without disorder, on the other hand, energy levels do cross each other, which results in an oscillating spin-density modulation at the sample boundary after the insertion of one flux quantum in the belt-shaped system. But the corresponding net spin transfer is only about one order of magnitude smaller than what is expected from the bulk SHC. These apparently contradictory results can be attributed to the violation of the spin conservation law in such a system. We also briefly address the dissipative Fermi surface contribution to spin polarization, which may be relevant to experimental measurements.
\end{abstract}

DOI: 10.1103/PhysRevB.72.235315

PACS number(s): 73.43.-f, 72.10.-d, 72.25.Dc

\section{INTRODUCTION}

Recently it has been proposed ${ }^{1,2}$ that in spin-orbit coupling (SOC) systems one may use an electric field to generate transverse spin currents in the absence of external magnetic fields. It has been argued ${ }^{1,2}$ that such a spin Hall effect (SHE) is intrinsic, contributed by all the electrons below the Fermi energy, and the corresponding spin currents are "dissipationless" as in contrast to the dissipative longitudinal charge currents which are only contributed by the electrons close to the Fermi energy and are strongly subjected to scattering effects.

The original proposals for the SHE are for the disorderfree cases. ${ }^{1,2}$ In the two-dimensional (2D) electron gas described by the Rashba model, ${ }^{2}$ it was shown based on a perturbative approach ${ }^{3,4}$ that the spin Hall conductance (SHC) is precisely cancelled by the vertex correction once the disorder is turned on. On the other hand, the vertex correction is found to vanish ${ }^{5}$ for the three-dimensional (3D) $p$-doped semiconductors described by the Luttinger model ${ }^{1}$ so that the SHC is still finite in the presence of weak disorder. Numerical calculations ${ }^{6-8}$ of the SHC seem to support such perturbative results of the distinct behavior for two models in the thermodynamic limit.

Experimentally the signatures of spin polarization have been observed recently in two-dimensional hole gas (2DHG) system ${ }^{9}$ and $3 \mathrm{D} n$-doped semiconductors, ${ }^{10}$ which have generated a lot of excitement concerning whether they are due to the intrinsic SHE mentioned above or some extrinsic effect. ${ }^{11}$ Bernevig and Zhang have shown that the vertex correction does vanish in the $2 \mathrm{DHG}^{12}$ and $3 \mathrm{D} n$-doped semiconductor ${ }^{13}$ systems. Furthermore, the mesoscopic SHE in the 2DHG is also found to be present based on the nonequilibrium Green function method ${ }^{14}$ and Landauer-Büttiker formula with attached leads, ${ }^{15}$ similar to (with larger magnitude than) the mesoscopic SHE found in the 2D Rashba model. ${ }^{16,17}$ But it is still unclear whether the bulk SHE in the 2DHG can survive in the thermodynamic limit beyond the perturbative approach.

In this paper, we perform numerical calculations for the 2DHG in the presence of disorder. We first show that the bulk SHC calculated from the Kubo formula is indeed robust against the disorder in extrapolation to the thermodynamic limit, which is consistent with the vertex correction calculation. ${ }^{12}$ It is also similar to the behavior for the Luttinger model, ${ }^{7}$ but is opposite to that of the 2D Rashba model. ${ }^{6}$ But when we perform a Laughlin's Gedanken "gauge experiment" on a belt-shaped sample to probe the spin transfer/accumulation due to the SHE, we obtain a null result due to the anticrossing between energy levels, which is quite similar to what has been previously seen in the $2 \mathrm{D}$ Rashba model. ${ }^{6}$ We point out that the absence of edge states in the 2DHG system causes the general level repulsion with the turn on of disorder, which leads to the disappearance of the net spin Hall accumulation in an open system. Furthermore, at zero disorder case, energy levels do cross each other and we find an oscillating spin-density modulation at the sample boundary after an adiabatic insertion of a flux quantum in the abovementioned Laughlin's gauge experiment. However, the corresponding net spin transfer is only about one order of magnitude smaller than what is expected from the calculated bulk SHC. We discuss the "conflicting" results of a finite SHC but without an intrinsic spin accumulation in the 2DHG, and point out that the underlying reason can be attributed to the violation of the spin conservation law in such a SOC system, where the SHC is no longer an unambiguous quantity for describing the spin transport. We also address the addition dissipative Fermi surface contribution to spin polarization, which may be relevant to experimental measurement. ${ }^{9,10}$

The remainder of the paper is organized as follows. In Sec. II, we numerically compute the SHC through the Kubo formula in a tight-binding model of the 2DHG system at different sample sizes and disorder strengths, and perform 
finite-size scaling analysis. In Secs. III A-III C, we perform a Laughlin's gauge experiment numerically to determine the intrinsic spin transfer/accumulation due to the adiabatic insertion of a magnetic flux quantum. And in Sec. III D, we compare the results with a system in a perpendicular external magnetic field and demonstrate the relationship between the intrinsic spin transfer (accumulation) and the existence of edge states. Finally, a summary is given in Sec. V.

\section{SPIN HALL CONDUCTANCE}

\section{A. Hamiltonian}

We start with the 2DHG Hamiltonian proposed by Bernevig and Zhang ${ }^{12}$

$$
H=\left(\gamma_{1}+\frac{5}{2} \gamma_{2}\right) \frac{k^{2}}{2 m}-\frac{\gamma_{2}}{m}(\vec{k} \cdot \vec{S})^{2}+\alpha(\vec{S} \times \vec{k}) \cdot \hat{z}
$$

For the convenience of the following numerical study, we further convert this continuum Hamiltonian into a tightbinding version on the square lattice. This may be realized by making the replacement $k_{\nu} \rightarrow \sin k_{\nu}$ and $k_{\nu}^{2} \rightarrow 2(1$ $-\cos k_{v}$ ). Two models are apparently equivalent near the band bottom where $k_{v} \rightarrow 0$. The resulting Hamiltonian reads

$$
\begin{aligned}
H= & -t \sum_{\langle i j\rangle}\left(c_{i}^{\dagger} c_{j}+c_{j}^{\dagger} c_{i}\right)+V_{L} \sum_{i \nu}\left(c_{i}^{\dagger} S_{\nu}^{2} c_{i+\hat{\nu}}+H . c .\right) \\
& +\frac{V_{L}}{4}\left(\sum_{i} c_{i}^{\dagger}\left\{S_{x}, S_{y}\right\}\left(c_{i+\hat{x}+\hat{y}}-c_{i+\hat{x}-\hat{y}}\right)+H . c .\right) \\
& +V_{R}\left(i \sum_{i} c_{i}^{\dagger} S_{y} c_{i+\hat{x}}-i \sum_{i} c_{i}^{\dagger} S_{x} c_{i+\hat{y}}+H . c .\right)-2 V_{L} \sum_{i} c_{i}^{\dagger} \\
& \times\left[\left(S_{x}^{2}+S_{y}^{2}\right)-\left(\frac{9}{4}-S_{z}^{2}\right)\left\langle k_{z}^{2}\right\rangle\right] c_{i}+4 t \sum_{i} c_{i}^{\dagger} c_{i}+\epsilon_{i} \sum_{i} c_{i}^{\dagger} c_{i},
\end{aligned}
$$

where the electron annihilation operator $c_{i}$ has four components characterized by the "spin" index $S_{z}=\frac{3}{2}, \frac{1}{2},-\frac{1}{2},-\frac{3}{2}$, respectively, and $i+\hat{\nu}(\hat{\nu}=\hat{x}, \hat{y})$ denote the nearest neighbors of site $i$. Here $t$ is the nearest-neighbor hopping integral defined as

$$
t=\frac{\hbar^{2}}{2 m a_{0}^{2}}\left(\gamma_{1}+\frac{5}{2} \gamma_{2}\right)
$$

where $m$ is the mass of the electron and $a_{0}$ is the lattice constant. $V_{L}$ and $V_{R}$ represents the strengths of the Luttingertype and Rashba-type spin-orbital couplings, respectively

$$
\begin{gathered}
V_{L} \equiv \frac{2 \gamma_{2}}{\gamma_{1}+\frac{5}{2} \gamma_{2}} t, \\
V_{R} \equiv \frac{\alpha}{\gamma_{1}+\frac{5}{2} \gamma_{2}} t .
\end{gathered}
$$

The parameters $\gamma_{1}$ and $\gamma_{2}$ in GaAs are given by $\gamma_{1}=6.92$ and $\gamma_{2}=2.1{ }^{19}$ so we have $t \sim 1.45 \mathrm{eV}$ and $V_{L} \sim 0.345 t$. We shall

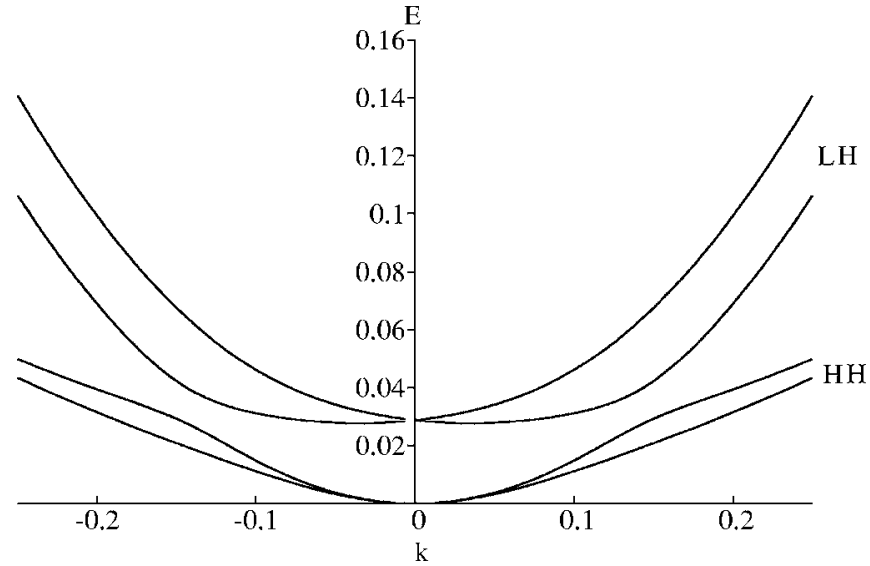

FIG. 1. Band structure for a clean 2DHG system determined by Eq. (2) (with $t=1$ ). The HH and LH denote the heavy hole band and light hole band, respectively.

choose $V_{R}=0.02 t$ for the Rashba-type coupling. By assuming that the 2DHG is confined in a well of the thickness $a$ $\sim 8.7 \mathrm{~nm}$, one approximately has $\left\langle k_{z}\right\rangle=0$ and $\left\langle k_{z}^{2}\right\rangle$ $\sim\left(\pi / a / a_{0}\right)^{2}$, where $a_{0} \sim 0.565 \mathrm{~nm}$ for GaAs. The corresponding energy spectrum for a clean sample $\left(\epsilon_{i}=0\right)$ is shown in Fig. 1. Due to the confinement in the $\hat{z}$ direction, a gap with $0.029 t-0.042 \mathrm{eV}$ opens up between the heavy hole $(\mathrm{HH})$ band and the light hole $(\mathrm{LH})$ band at $\Gamma$ point (see Fig. 1). For simplicity, we shall set $t=1$ in the rest of paper. Finally, $\epsilon_{i}$ in Eq. (2) accounts for the on-site and spinindependent disorder strength, which is randomly distributed within $[-W / 2, W / 2]$.

\section{B. Spin Hall conductance}

The SHC is defined by the Kubo formula ${ }^{18}$

$$
\sigma_{S H}=\frac{2}{N}\left\langle\operatorname{Im} \sum_{E_{n}<E_{f}<E_{m}} \frac{\left\langle n\left|j_{x}^{z \operatorname{spin}}\right| m\right\rangle\left\langle m\left|j_{y}\right| n\right\rangle}{\left(E_{m}-E_{n}\right)^{2}}\right\rangle,
$$

where $N$ is the total number of lattice sites, $E_{f}$ denotes the Fermi energy, $E_{m}\left(E_{n}\right)$ is the eigen energy, and $\langle\ldots\rangle$ is averaged over all disorder configurations. The charge current operator $j_{\mu}$ and spin current operator $j_{\mu}^{\nu \text { spin }}$ are defined as

$$
\begin{gathered}
j_{\mu} \equiv e v_{\mu}, \\
j_{\mu}^{\nu \text { spin }} \equiv \frac{1}{2}\left\{v_{\mu}, S_{\nu}\right\},
\end{gathered}
$$

where the velocity operator $\mathbf{v}$ is the conjugate operator of the position operator $\mathbf{R} \equiv \sum_{i \sigma} \mathbf{r}_{i} n_{i \sigma}$ ( $n_{i \sigma}$ is the electron number operator at site $i$ with spin index $\sigma$ ), defined by the standard relation

$$
v_{\mu}=\frac{i}{\hbar}\left[H, R_{\mu}\right]
$$

For a pure sample $\left(\epsilon_{i}=0\right)$, the Hamiltonian can be easily diagonalized in momentum space, and we can determine $\sigma_{S H}$ vs $E_{f}$ for a very large lattice $\left(N=L^{2}\right.$ with $\left.L=1000\right)$ as shown 


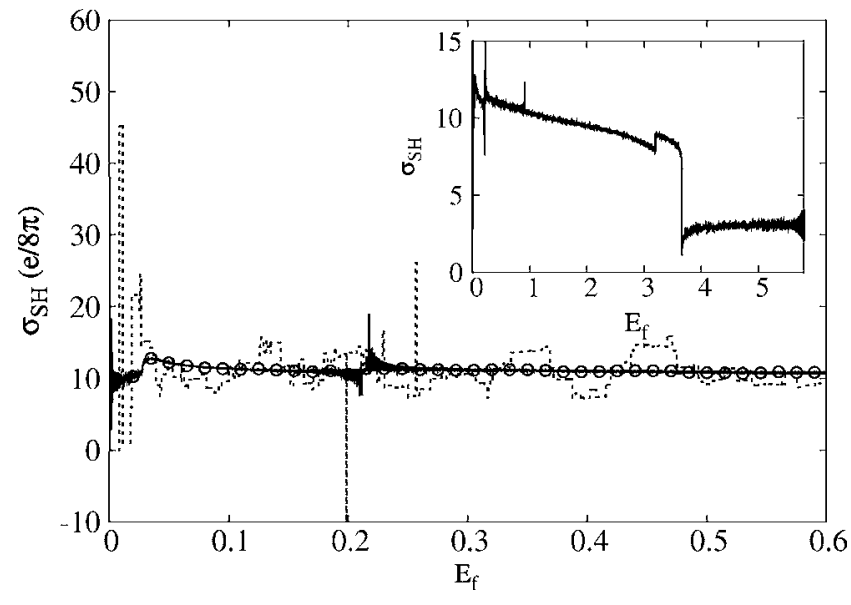

FIG. 2. $\sigma_{S H}$ vs $E_{f}$ at $W=0$. The inset shows the whole Fermi energy region and the main panel shows the details around the band bottom. The solid line is at $1000 \times 1000$ lattice, the dashed line is at $24 \times 24$ lattice, and the open circles are at $24 \times 24$ lattice with averaged over $1000 \mathrm{BCs}$.

in the inset of Fig. 2. Since the tight-binding model is a good approximation of the original model only when $E_{f}$ is near the band bottom, we shall focus on the regime of $0<E_{f}<0.6$ as represented in the main panel of Fig. 2 by the solid curve. One can see that $\sigma_{S H}$ in this region is a little bit larger than $10 \mathrm{e} / 8 \pi$ and very flat as a function of $E_{f}$ except for that within the gap $(\sim 0.029)$ between the HH and LH bands.

Because the momentum is no longer a good quantum number in the presence of disorder, Eq. (2) cannot be diagonalized in the momentum space when $\epsilon_{i} \neq 0$. In the latter case, the maximal lattice size reachable by the numerical calculation is usually much smaller than $L=1000$ (around $L=20-30$ ). To check what happens at smaller lattices, we compute $\sigma_{S H}$ on an $L=24$ lattice in the pure system with a periodic boundary condition $(\mathrm{BC})$ and the result is illustrated by the dotted curve in Fig. 2 . The corresponding $\sigma_{S H}$ exhibits very large fluctuations whose magnitude can be several times larger than the converged value at $L=1000$. To remove such finite-size fluctuations, we introduce an average over different BCs. A general (twisted) BC is given by

$$
\begin{aligned}
& \psi(x+L, y)=e^{i \theta_{x}} \psi(x, y), \\
& \psi(x, y+L)=e^{i \theta_{y}} \psi(x, y),
\end{aligned}
$$

where $\theta_{\mu}$ 's are defined within $[0,2 \pi]$ with $\theta_{\mu}=0$ or $2 \pi(\mu$ $=x, y$ ) corresponding to the periodic BC. Since the $\mathrm{SHC}$ should not be sensitive to the BCs in the thermodynamic limit, in principle, $\sigma_{S H}$ in Eq. (4) can be always defined as averaged over both disorder and twisted BCs in such a limit. For a clean system, the averaged $\sigma_{S H}$ at $L=24$ over 1000 randomly generated $\mathrm{BCs}$ is shown by the open circles in Fig. 2. One can clearly see that the large fluctuations in $\sigma_{S H}$ at $L=24$ (dotted curve) have been significantly reduced after the $\mathrm{BC}$ averaging, and the result (open circles) coincides with $\sigma_{S H}$ obtained at $L=1000$ (solid line) very well. It indicates that the finite-size effect at smaller size $(L=24)$ can be properly removed by the $\mathrm{BC}$ average. As a consequence, the

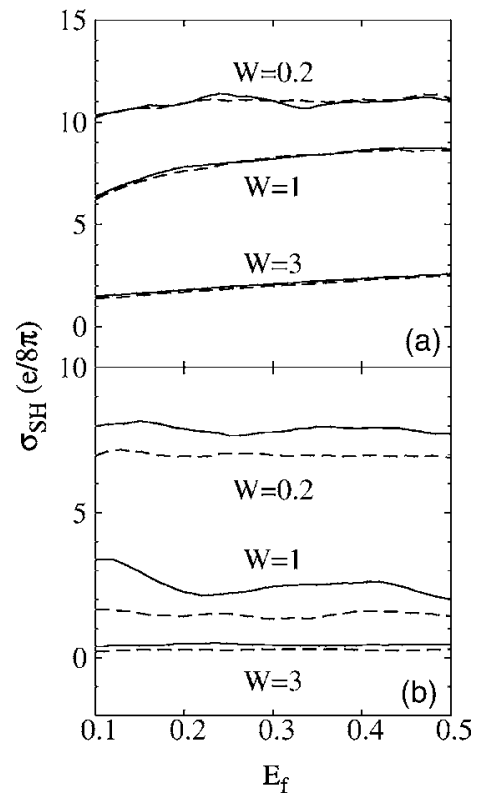

FIG. 3. $\sigma_{S H}$ vs $E_{f}$ at $W=0.2,1$, and 3. The solid curves are for $L=16$, and the dashed ones for $L=24$; (a) is for the 2DHG system and (b) is for the spin-3/2 Rashba model by setting $V_{L}=0$.

$\mathrm{BC}$ averaged SHC behaves much smoother than at a fixed $\mathrm{BC}$, and is thus more suitable for a finite-size scaling analysis. We note that the $\mathrm{BC}$ average is also required by the gauge-invariant condition for SHC in a finite-size system. When we apply an electric field through a time-dependent vector potential, it acts as a generalized boundary phase evolving with time (see below). Thus the SHC averaged over time is gauge invariant and equivalent to the $\mathrm{BC}$ averaged SHC. ${ }^{6}$ Such a numerical method has been previously used in the study of the 2D Rashba model and 3D Luttinger model. ${ }^{6,7}$ Similar boundary phase averaged charge Hall conductance can be also related to a topological invariant Chern number. ${ }^{18}$

Now we use the above method to study the sample-size dependence of $\sigma_{S H}$ at $W=0.2$ for weak disorder strength; $W=1$ for intermediate disorder; and $W=3$ for strong disorder. The calculated $\sigma_{S H}$ is presented in Fig. 3(a) for two sample sizes: $L=16$ [averaged over 20000 configurations (solid line)] and $L=24$ [averaged over 5000 configurations (dashed line)]. The size dependence of $\sigma_{S H}$ is very weak for all three cases at different $E_{f}$ 's. For comparison, we have also done a similar calculation for a "spin-3/2 (linear) Rashba model," which corresponds to Eq. (2) at $V_{L}=0$ and $V_{R}=0.5$. SHC at $W=0.2,1$, and 3, with $L=16$ and 24, respectively, are shown in Fig. 3(b), which clearly decrease with the increase of the sample length, similar to the result in the spin-1/2 Rashba model. ${ }^{6}$

To further investigate the effects of the lattice size and disorder, we calculate the averaged $\sigma_{S H}$ within a chosen energy interval $[0.3,0.4]$ at various disorder strengths and sample sizes as shown in Fig. 4. $\sigma_{S H}$ is almost independent of sample sizes and reduces monotonically with increasing $W$, which is different from the spin-1/2 Rashba model but similar to the behavior in the 3D Luttinger model. ${ }^{7}$ In the weak disorder limit, $\sigma_{S H}$ approaches the value slightly larger 


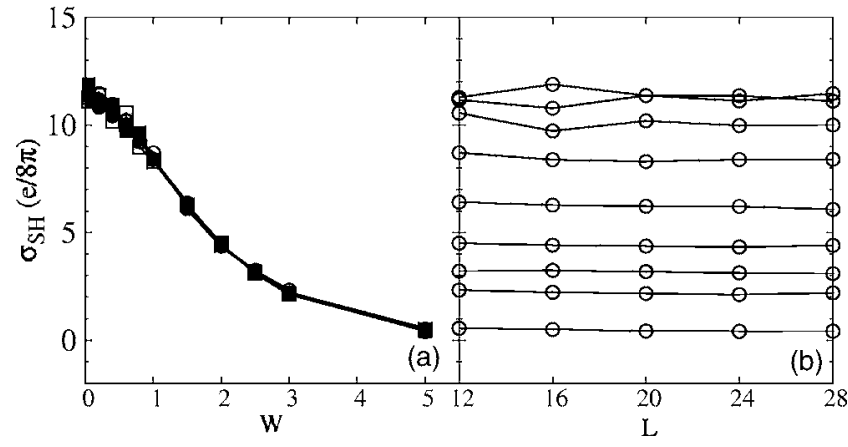

FIG. 4. $W$ and sample-size dependence of $\sigma_{S H}$ with $E_{f}$ averaged within the interval $[0.3,0.4]$. (a) $\sigma_{S H}$ vs $W$. The open squares are for $L=12$, close squares for $L=16$, open circles for $L=20$, and close circles for $L=24$. (b) $\sigma_{S H}$ vs $L$. The lines from top to bottom are for $W=0.05,0.2,0.6,1,1.5,2,2.5,3$, and 5 , respectively.

than $10 \mathrm{e} / 8 \pi$, which is quite close to the value $(\sim 9 e / 8 \pi)$ obtained $^{20}$ in an analytic calculation in a different model for the 2DHG system. Thus we establish that SHC is finite extrapolating to thermodynamic limit until the disorder strength reaches a critical value $W_{c}=5.0$.

\section{INTRINSIC SPIN TRANSFER AND ACCUMULATION}

In the last section, we have shown the numerical evidence that a finite $\sigma_{S H}$ can survive in the presence of disorder with the sample size being extrapolated to the thermodynamic limit. This is in sharp contrast to the 2D Rashba model in which $\sigma_{S H}$ vanishes in the thermodynamic limit with the turn on of very weak disorder strength. ${ }^{6}$ Such a distinct behavior in $\sigma_{S H}$ is consistent with the perturbation theories in which the vertex correction also results in opposite conclusions on the fate of $\sigma_{S H}$ in two models, as mentioned in the Introduction.

In general, a finite conductance should lead to an accumulation of current carriers at the edges (open boundaries) of the sample according to the current conservation law. However, in the SOC system the spin current conservation law does not exist as the spin is not conserved. Thus the relation between the SHC and the spin accumulation is not straightforwardly present.

In this section, we will try to probe the spin transfer or accumulation effect directly by performing numerically a Laughlin's gauge experiment, which was first used in the IQHE system ${ }^{21,22}$ to explain why an integer number of charges can be transported across the sample in the transverse direction when a longitudinal electric field is applied via an adiabatic flux insertion process. This method has been recently generalized to the SOC system by Sheng et al. ${ }^{6}$ in the study of the SHE in the 2D Rashba model.

\section{A. Gauge experiment and level crossing}

The numerical gauge experiment will be performed on a belt-shaped sample with a magnetic flux $\Phi$ adiabatically inserted at the center of the ribbon [see Fig. 5(a)]. Such a belt-shaped geometry with the magnetic flux can be implemented in a square lattice sample shown in Fig. 5(b) by
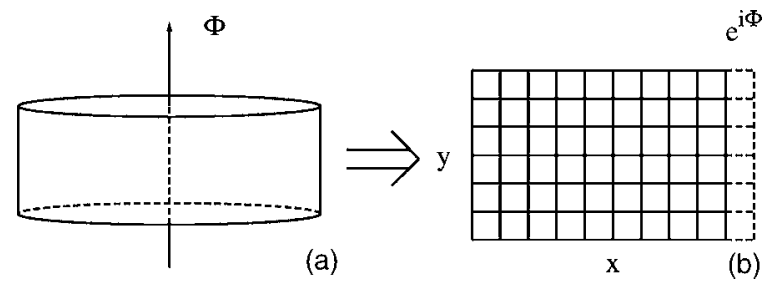

FIG. 5. (a) The geometry of the sample in the gauge experiment. It is like a belt enclosing a flux in the center. (b) The implementation of (a) in the numerical calculation. The dashed line with a phase factor $e^{i \Phi}$ along the $\hat{x}$ axis means a twist $\mathrm{BC}$, which represents the flux in (a).

imposing a twisted $\mathrm{BC}$ along the $\hat{x}$ direction and an open $\mathrm{BC}$ along the $\hat{y}$ direction. Namely, with a gauge transformation, one may impose the flux $\Phi$ by changing the BC along the $\hat{x}$ direction from the periodic $\mathrm{BC}$ to a twist $\mathrm{BC}$ with the phase twist $\theta_{x}=\Phi$ indicated by the dashed line and the phase factor $e^{i \Phi}$ in Fig. 5(b).

Numerically the energy spectrum of single electron states at a fix $\Phi$ can be calculated in the geometry of Fig. 5(b). Figure 6 shows such calculated energy spectra as a function of $\Phi$ in the pure system. Note that the system is equivalent at $\Phi=0$ and $\Phi=2 \pi$ as the Hamiltonian is periodic $H(\Phi=0)$ $=H(\Phi=2 \pi)$, and the energy spectrum is symmetric between $0 \leqslant \Phi \leqslant \pi$ and $\pi \leqslant \Phi \leqslant 2 \pi$ due to a time-reversal symmetry, such that only the first half is plotted in Fig. 6 .

One may imagine changing the flux $\Phi$ adiabatically from 0 to $2 \pi$ to follow the corresponding change of the system. Generally there are two possibilities for the evolution of the ground state with the electrons filled below the Fermi energy $E_{f}$. If each single electron state always anti crosses with other states (level repulsion), it will eventually return to the original state after the insertion of one flux quantum of $\Phi$ $=2 \pi$ sufficiently slow (i.e., adiabatically such that there is no quantum transition to other levels during this operation); on the other hand, if a level crossing occurs for single electron states during the adiabatical increase of $\Phi$, then the original

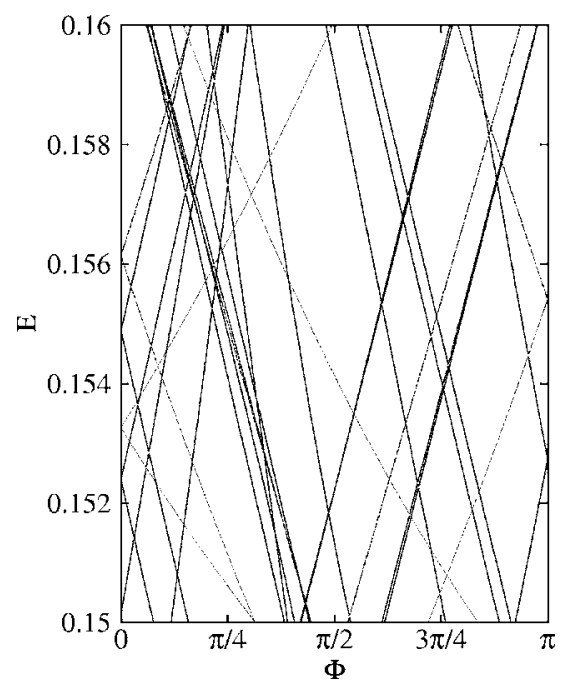

FIG. 6. The evolution of the single electron state energy with $\Phi$ for the $2 \mathrm{DHG}$ on a $32 \times 32$ clean sample $(W=0)$. 


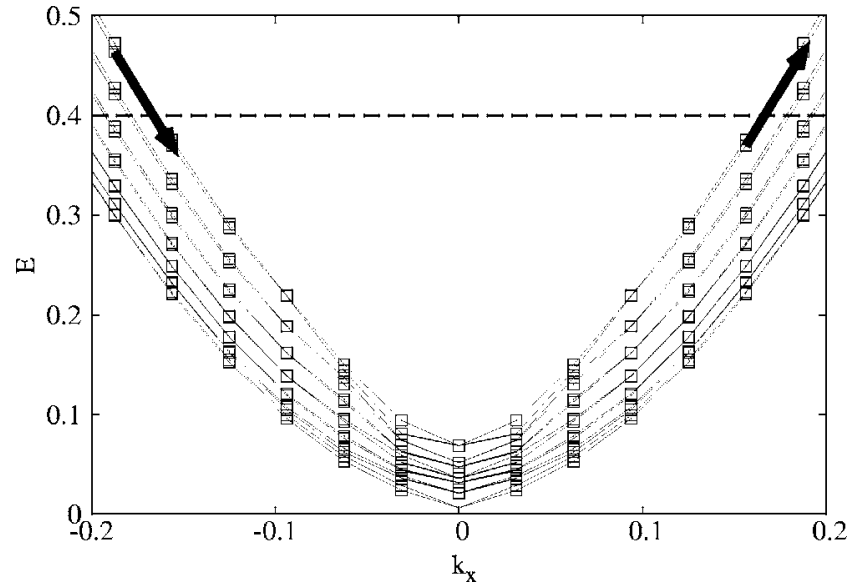

FIG. 7. The miniband structure of a $32 \times 32$ clean sample. Two arrows represent the evolution of two states by tuning the parameter $\Phi$, which starts from the beginning at $\Phi=0$ and arrives at the end of the arrows at $\Phi=2 \pi$. The dashed line indicates the Fermi energy.

ground state generally will evolve into a different state through the change of the single state occupation number, even though the single electron energy spectrum must remain the same after the insertion of one flux quantum.

A slow increase of the flux $\Phi$ is equivalent to applying a weak electric field along the $\hat{x}$ direction

$$
\epsilon_{x}=-\frac{1}{L_{x}} \frac{\partial \Phi}{\partial t}
$$

Then the intrinsic charge/spin Hall effect due to an electric field in belt-shaped system will lead to the transfer of charge/ spin along the $\hat{y}$ axis during the flux insertion. If there is to be charge/spin accumulation at the open edges [Fig. 5(a)], it means that the ground state should not return to the original one after the adiabatic flux insertion, which is the essence of Laughlin's gauge experiment for the IQHE system. Therefore, the level crossing of single electron states serves as a necessary condition for the existence of intrinsic spin transfer/accumulation for a SHE system. As clearly shown in Fig. 6, which is determined on an $L=32$ lattice, the single electron energy levels do simply cross in the pure $2 \mathrm{DHG}$ system. The resulting net spin transfer will be analyzed in the following section.

\section{B. Spin Transfer at $W=0$}

In this section, we calculate the spin transfer induced by tuning the flux $\Phi$ from 0 to $2 \pi$ in the pure case $\left(\epsilon_{i}=0\right)$. Starting from the periodic BC $(\Phi=0)$, the momentum $k_{x}$ along $\hat{x}$ direction is a good quantum number. And due to the open boundary along the $\hat{y}$ direction, the electron states form a mini-band structure which is shown in Fig. 7 and can be denoted as $\left|k_{x}, n\right\rangle$, where $n$ is the index of the mini bands. If we choose an arbitrary Fermi energy $E_{f}$, the ground state corresponds to that all the single particle states below $E_{f}$ are occupied, whereas unoccupied above $E_{f}$. So the spin density along the $\hat{y}$ direction (labeled by $y \equiv j_{y}$ ) at the initial state can be defined by

$$
S_{z}^{i}(y) \equiv \sum_{E_{k_{x}, n}<E_{f}}\left\langle k_{x}, n\left|\sum_{j_{x}} c_{j}^{+} S_{z} c_{j}\right| k_{x}, n\right\rangle .
$$

When one inserts the flux adiabatically from zero to $\Phi$, the state $\left|k_{x}, n\right\rangle$ will evolve to $\left|P_{x}=k_{x}+\Phi / L_{x}, n\right\rangle$. For $k_{x}>0$ and $<0$, the corresponding energies of the states will increase and decrease, respectively, which is illustrated by arrows in Fig. 7. Thus one finds a simple level crossing for two levels close to each other with opposite signs of $k_{x}$, which represents the level crossing shown in Fig. 6 for pure system due to no mixing term between different states in the Hamiltonian. After the insertion of one flux quantum, the state $\left|k_{x}, n\right\rangle$ evolves into a new state $\left|k_{x}+2 \pi / L_{x}, n\right\rangle$ at $\Phi=2 \pi$. Then the spin density at the final state is given by

$$
S_{z}^{f}(y)=\sum_{E_{k_{x}, n}<E_{f}}\left\langle k_{x}+\frac{2 \pi}{L_{x}}, n\left|\sum_{j_{x}} c_{j}^{+} S_{z}^{j} c_{j}\right| k_{x}+\frac{2 \pi}{L_{x}}, n\right\rangle .
$$

The resulting change in the spin density due to threading a flux quantum is $\Delta S_{z}(y)=S_{z}^{f}(y)-S_{z}^{i}(y)$.

$\Delta S_{z}(y)$ in a pure $2 \mathrm{DHG}$ with $500 \times 100$ lattice is shown in the inset of Fig. 8(a), where the Fermi energy is $E_{f}=0.4$. We recall that due to the time-reversal invariance, $S_{z}^{i}(y)=0 \mathrm{ev}-$ erywhere. After adding the $2 \pi$ flux quantum, the spin density shows some strong peaks at the boundaries, whereas with a much weaker magnitude in the bulk. We show the spinwave-like modulation in $\Delta S_{z}(y)$ near one edge of the beltshaped sample at various lattice sizes in the main panel of Fig. 8(a). Here $\Delta S_{z}(y)$ is almost sample-size independent at the edge, whereas it reduces monotonically with the sample size in the bulk. So in the thermodynamic limit, there should be only the spin density modulation present near the edge. Similar results with smaller magnitude are also obtained for an electron spin-1/2 Rashba model as shown in Fig. 8(b) for comparison. Such a spin density modulation does support a net spin transfer along the $\hat{y}$ direction, accompanying an electric field generated along the $\hat{x}$ direction due to the inserting flux according to Eq. (7), but the magnitude of the total (integrated) $\Delta S_{z} \sim 0.2 \hbar$ at one edge is much less than one spin quantum, which is in sharp contrast to the large bulk SHC [the calculated $\sigma_{S H} \sim 11 e / 8 \pi$, that suggests a total spin transfer of 5.5( $\hbar / 2)$ spin quanta]. This large discrepancy between two approaches indicates that the SHC is no longer an unambiguous quantity for measuring the spin transport even in the pure case. In the following we further explore the disorder case.

\section{Disorder effect}

Now we consider the disorders effect. Figure 9 shows the results for a chosen disorder configuration with $W=0.05$ in a $32 \times 32$ lattice. A careful examination of the energy levels reveals that each eigenstate goes up and down, making several large angle turns due to backward scattering. These energy levels never cross with each other, except for at $\Phi=0$ and $\pi$, where two levels become exactly degenerate (Kramers degeneracy). Then if one follows any Kramers degenerated pair of states starting from $\Phi=0$ to $\Phi=2 \pi$, one will always go back to exactly the same pair of states. Namely, 


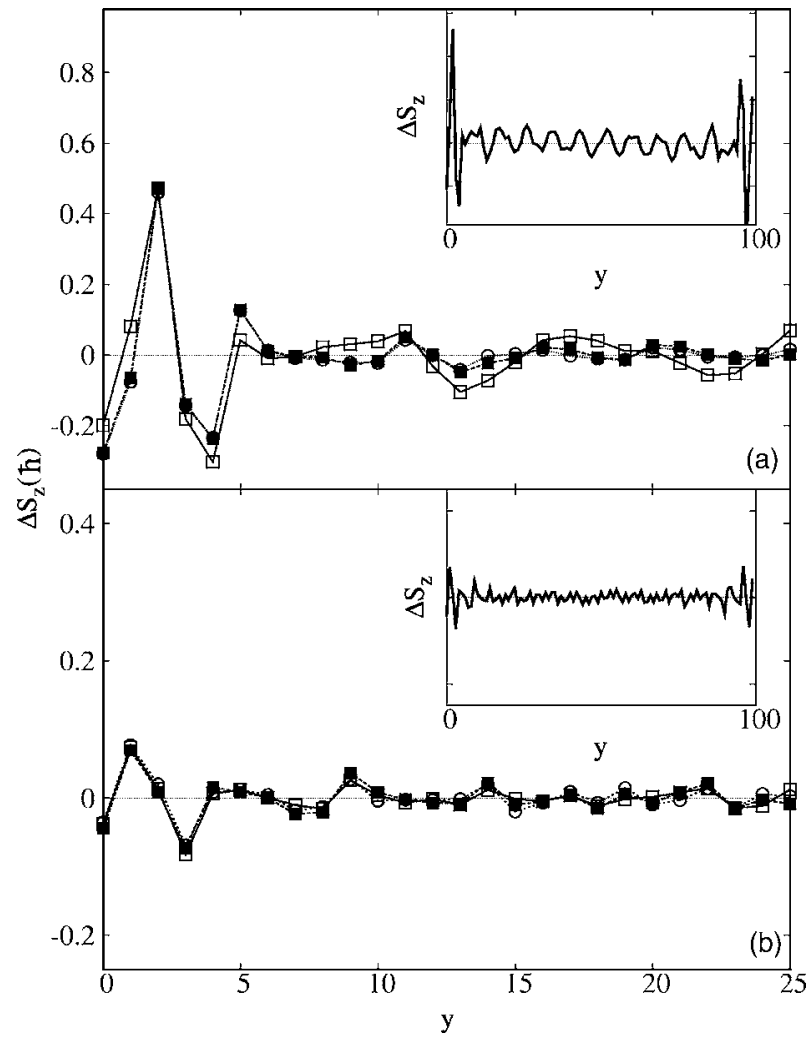

FIG. 8. The spin density changes between the state at the beginning and the end of the procedure of increasing the flux from 0 to $2 \pi$ in the pure samples. The main panel shows the data around one edge for various lattice sizes, while the inset shows the whole result on a $500 \times 100$ lattice. The Fermi energy is fixed at $E_{f}=0.4$; (a) is for the 2DHG. The solid lines with open squares are for 1000 $\times 100$ lattice, the dashed lines with close squares are for 500 $\times 500$ lattice, and the dotted lines with open circles are for 1000 $\times 1000$ lattice; (b) is for the 2D Rashba model. The solid lines with open squares are for $100 \times 100$ lattice, the dashed lines with close squares are for $500 \times 100$ lattice, and the dotted lines with open circles are for $1000 \times 1000$ lattice.

after the adiabatic insertion of one flux quantum, all states evolve exactly back to the starting states.

For comparison with the $W=0$ case, in Fig. 10(a) we combine an enlarged plot of two adjacent energy levels in Fig. 10 together with the pure case, where the solid lines are for the disordered case and the dashed lines are for the clean case. In the pure case, an electron at the state $\left|i_{1}\right\rangle\left(\left|i_{2}\right\rangle\right)$ will finally evolve to $\left|f_{1}\right\rangle\left(\left|f_{2}\right\rangle\right)$ with increasing flux due to the level crossing. However, in contrast to the level crossing in the pure system, two energy levels, $\left|i_{1}\right\rangle,\left|i_{2}\right\rangle$, in the disordered system generally show a level repulsion as one increases the flux $\Phi$, to evolve into the final states of $\left|f_{2}\right\rangle$ and $\left|f_{1}\right\rangle$, respectively [see Fig. 10(a)]. The level repulsion in the disordered case represents a scattering process shown in Fig. 10(b).

We have done similar calculations at different lattice sizes, such as $8 \times 8,16 \times 16$, and $24 \times 24$. The results are all similar except that the "gaps" characterizing the level repulsion decrease with the lattice size, which are roughly proportional to $1 / N$. According to the discussion in Sec. III A, the

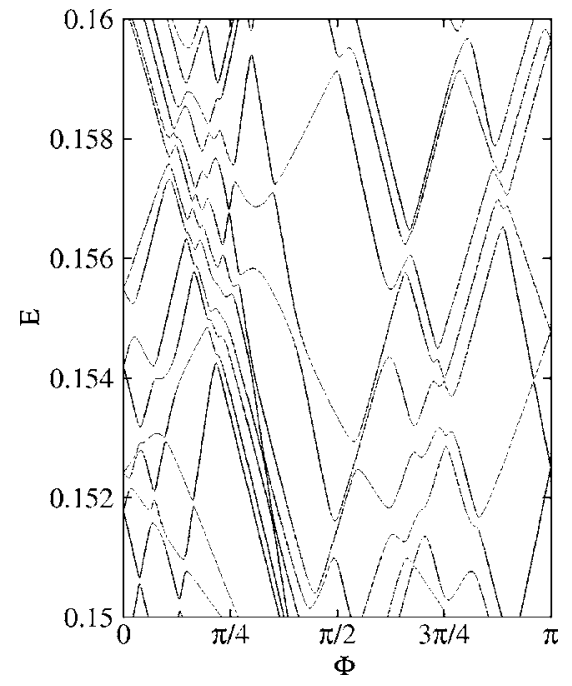

FIG. 9. The evolution of the single electron states with $\Phi$ for the 2 DHG on $32 \times 32$ lattice for a disordered sample with $W=0.05$.

adiabatic insertion of the flux $\Phi$ will not generate true spin transfer or accumulation as the ground state simply evolves back after a flux quantum insertion. In fact, we have checked various weak disorder strengths and always found the same generic result.

Therefore, we conclude that there is no intrinsic spin Hall accumulation (spin transfer) for the $2 \mathrm{DHG}$ system in the disorder case. This conclusion is in sharp contrast to the calculated SHC at small $W$ in Sec. II, which remains finite and close to the value of the pure case when the disorder is weak. According to the discussion at the beginning of Sec. III, these two approaches, for a bulk system on a torus or as an open system with two edges, are not necessarily in accordance with each other, because the spin current continuity equation is broken. For example, a backward scattering of an electron by an impurity shown by Fig. 10(b) can simultaneously change the sign of its momentum as well as its spin direction due to the SOC. Then one can find that the spin current is still additive (same sign) in this scattering process, which contributes to the SHC accordingly, but there should be no true spin transfer taking place because of the backward scattering of the spin carrier electron which moves back in direction after the scattering.

\section{Edge state}

To further understand the condition for the presence of level crossing in the disordered case, we apply an external magnetic field perpendicular to the 2D sample (with a flux strength $\pi / 8$ per plaquette) and repeat the above calculation. We find that most of the gaps still behave as $1 / N$, similar to those in the nonmagnetic-field case. These are bulk states. But we can also identify a few levels whose level-repulsion gaps reduce exponentially with $N$ with the increase of the sample size. For example, such kind of gap at $32 \times 32$ lattice size is about $1 / 30$ of the one at $16 \times 16$ lattice size, an exponential dependence on system length. We show some of these "level crossings" in Fig. 11 with the same parameters 


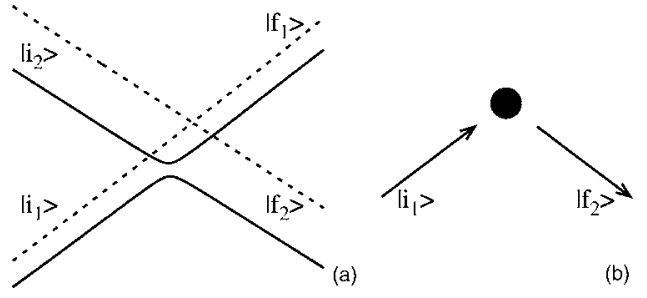

FIG. 10. (a) The comparison of the evolutions of two levels for clean system (dashed line) and disordered system with $W=0.05$ (dashed line). The levels are crossing at clean system and anticrossing at disorder system. The $\left|i_{1}\right\rangle$ and $\left|i_{2}\right\rangle$ are the initial states while the $\left|f_{1}\right\rangle$ and $\left|f_{2}\right\rangle$ are the final states. (b) The process of a state $\left|i_{1}\right\rangle$ scattered to $\left|f_{2}\right\rangle$ by the disorder. Such a process is leading to the anti-crossing as shown in (a).

as in Fig. 9. The gaps in Fig. 11 are much smaller than the ones in Fig. 9 and hard to distinguish with the naked eye. The exponential decaying form of the gaps indicate the existence of level crossings in the thermodynamic limit in the presence of a perpendicular magnetic field, which corresponds to an IQHE. Then a net charge (spin) is transported from one edge to the other correspondingly. ${ }^{6}$

We have also systematically compared the spatial distribution of electron density $\rho_{i}=\left\langle c_{i}^{\dagger} c_{i}\right\rangle$ of the states in the 2DHG system with and without the presence of a perpendicular magnetic field. It is found that the level crossing only occurs for the edge states. Physically this is because in the thermodynamic limit the distance between two edge states in the opposite sides of the sample goes to infinity, which makes the scattering between them vanish exponentially. Thus the absence of level crossing (and the intrinsic spin Hall transfer) in the 2DHG system can be attributed to the fact that there do not exist any edge states in such a system in the absence of perpendicular external magnetic fields.

\section{CONCLUSIONS AND DISCUSSIONS}

In the present work, we have numerically calculated the SHC through the Kubo formula at different lattice sizes and impurity strengths. A reasonable finite-size scaling analysis shows that the bulk SHC remains finite in the thermodynamic limit in the disordered 2DHG up to a critical disorder strength $W_{c}=5$. This result is consistent with the perturbative calculation based on the vertex correction. ${ }^{12}$

However, we have also found the strong evidence that the net spin Hall accumulation or spin transfer disappears in the disordered case based on a numerically performed Laughlin's gauge experiment. We have interpreted the conflicting results of the SHC and direct spin accumulation calculations as due to the fact that the spin is not conserved in an SOC system and there is no longer a conservation law to govern the spin current. Therefore the SHC is no more a unique quantity to characterize the spin transport, where electron spin can relax and vanish in the bulk without being transported across the sample by a transverse electric field.

By comparing with the case in the presence of an applied perpendicular magnetic field, which is in the IQHE regime,

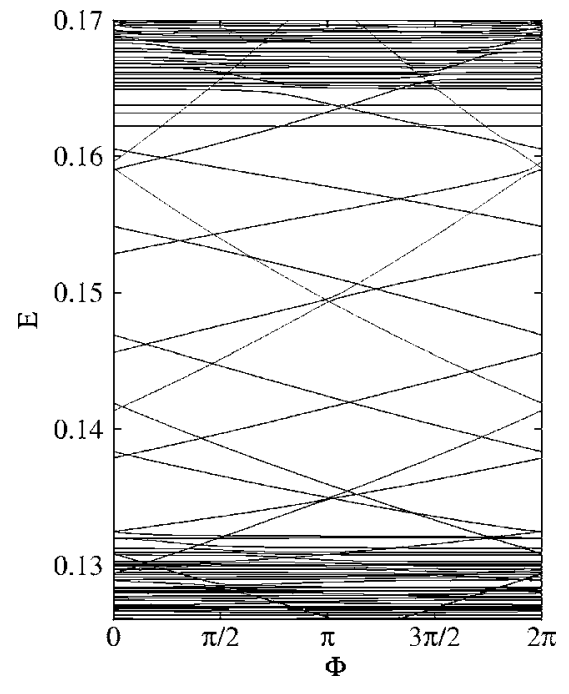

FIG. 11. The evolution of the single electron states with $\Phi$ in the system with an external magnetic field on a $32 \times 32$ lattice. The strength of the magnetic field is $\pi / 8$ per plaquette.

we have shown that the absence of an intrinsic spin transfer (accumulation) can be related to that there are no edge states in the 2DHG system, in contrast to the IQHE case.

We further address the validity of the above adiabatic argument in the thermodynamic limit. It is noted that since an intrinsic spin transfer (accumulation) is always absent in a finite size system according to the above argument, it is very hard to imagine that such an effect could be restored as an intrinsic one by the Landau-Zener tunneling ${ }^{8}$ between two anti-crossing levels in the thermodynamic limit. Of course, the Landau-Zener tunneling between the level repulsion gaps in the large sample size limit (the level repulsion gap vanishes in a $1 / N$ fashion, with the same rate as the average level spacing vanishing in this limit) can still contribute to dissipative transport currents, and similar to the conventional charge transport, one may expect a dissipative spin transport term appearing, besides the null intrinsic spin transfer. Here the dissipative spin transport is qualitatively different from the true level crossing with edge states. At a finite $W$, in the longitudinal channel, the so-called "Thouless conductance" term can be obtained based on the sensitivity of the energy level to the threading flux, giving rise to a finite $\sigma_{x x}{ }^{23}$ which is subject to the detailed scattering mechanism as contributed by the levels near the Fermi energy. In the present SOC system, a dissipative spin Hall transfer related to this longitudinal conductance is indeed possible, which similarly involves only the states near the Fermi energy. We note that such a dissipative term may also be important for the spin polarization measured experimentally. ${ }^{9,10}$

The vanishing spin transfer revealed through Laughlin's Gedanken gauge experiment for the present 2DHG and 2D electron Rashba model ${ }^{6}$ under an adiabatic procedure may be a generic behavior for a metallic system. ${ }^{24}$ It would be interesting to directly probe this property for more SOC systems, including the 3D Luttinger model, although numerically it is more challenging for the latter as it is much harder to do the finite-size scaling for the level repulsion gaps in a 3D sys- 
tem. Finally, it is interesting to note that several recent papers have studied topological SHE in insulating electron systems with SOC. ${ }^{25}$ In these models, it becomes clear that bulk intrinsic SHC can result in spin transfer and accumulation, with the presence of spin-polarized edge states, which is associated with a topological invariant. This is in agreement with our general conclusion that the intrinsic spin transfer relies on the existence of current-carrying edge state.

\section{ACKNOWLEDGMENTS}

We would like to thank F. D. M. Haldane, S. C. Zhang, and $\mathrm{M}$. W. Wu for insightful discussions. This work is supported by NSFC Grant Nos. 10374058 and 90403016 (Z.Y.W.), ACS-PRF 41752-AC10, the NSF grant/DMR0307170 (D.N.S.). The computation of this project was partially performed on the HP-SC45 Sigma-X parallel computer of ITP and ICTS, CAS.
${ }^{1}$ S. Murakami, N. Nagaosa, and S. C. Zhang, Science 301, 1348 (2003).

${ }^{2}$ J. Sinova et al., Phys. Rev. Lett. 92, 126603 (2004).

${ }^{3}$ J. I. Inoue, G. E. W. Bauer, and L. W. Molenkamp, Phys. Rev. B 70, 041303(R) (2004).

${ }^{4}$ E. G. Mishchenko, A. V. Shytov, and B. I. Halperin, Phys. Rev. Lett. 93, 226602 (2004).

${ }^{5}$ S. Murakami, Phys. Rev. B 69, 241202(R) (2004).

${ }^{6}$ D. N. Sheng, L. Sheng, Z. Y. Weng, and F. D. M. Haldane, condmat/0504218.

${ }^{7}$ W. Q. Chen, Z. Y. Weng, and D. N. Sheng, cond-mat/0502570, Phys. Rev. Lett. (to be published).

${ }^{8}$ K. Nomura, J. Sinova, N. A. Sinitsyn, and A. H. MacDonald, cond-mat/0506189; K. Nomura, J. Sinova, T. Jungwirth, Q. Niu, and A. H. MacDonald, Phys. Rev. B 71, 041304(R) (2005).

${ }^{9}$ J. Wunderlich, B. Käestner, J. Sinova, and T. Jungwirth, Phys. Rev. Lett. 94, 047204 (2005).

${ }^{10}$ Y. K. Kato, R. C. Myers, A. C. Gossard, and D. D. Awschalom, Science 306, 1910 (2004).

${ }^{11}$ H.-A. Engel, B. I. Halperin, and E. I. Rashba, cond-mat/0505535 (unpublished).

${ }^{12}$ B. A. Bernevig and S. C. Zhang, cond-mat/0411457 (unpublished).

${ }^{13}$ B. A. Bernevig and S. C. Zhang, cond-mat/0412550 (unpublished).
${ }^{14}$ S. Y. Liu and X. L. Lei, cond-mat/0503352 (unpublished).

${ }^{15} \mathrm{M}$. W. Wu and J. Zhou, cond-mat/0503616 (unpublished).

${ }^{16}$ L. Sheng, D. N. Sheng, and C. S. Ting, Phys. Rev. Lett. 94, 016602 (2005); B. K. Nikolié, L. P. Zârbo, and S. Souma, condmat/0408693 (unpublished).

${ }^{17}$ E. M. Hankiewicz, L. W. Molenkamp, T. Jungwirth, and J. Sinova, Phys. Rev. B 70, 241301(R) (2004).

${ }^{18}$ Q. Niu, D. J. Thouless, and Y. S. Wu, Phys. Rev. B 31, 3372 (1985).

${ }^{19}$ See, Quantum Theory of the Optical and Electronic Properties of Semiconductors, H. Haug and S. W. Koch (World Scientific, Singapore, 1990).

${ }^{20}$ J. Schliemann and D. Loss, Phys. Rev. B 71, 085308 (2005).

${ }^{21}$ R. B. Laughlin, Phys. Rev. B 23, 5632(R) (1981).

${ }^{22}$ B. I. Halperin, Phys. Rev. B 25, 2185 (1982).

${ }^{23}$ T. Ando, Phys. Rev. B 40, 5325 (1989).

${ }^{24}$ F. D. M. Haldane, Phys. Rev. Lett. 93, 206602 (2004).

${ }^{25}$ L. Kane and E. J. Mele, cond-mat/0411737 (unpublished); condmat/0506581 (unpublished); L. Sheng, D. N. Sheng, C. S. Ting, and F. D. M. Haldane, cond-mat/0506589 (unpublished); B. A. Bernevig and S. C. Zhang, cond-mat/0504147 (unpublished); X.-L. Qi, Y.-S. Wu, and S. C. Zhang, cond-mat/0505308 (unpublished). 